\title{
LÓPEZ LUJÁN Leonardo y Guilhem OLIVIER (eds), El sacrificio humano en la tradición religiosa mesoamericana
}

Nathalie Ragot

\section{(2) OpenEdition}

\section{Journals}

Édition électronique

URL : https://journals.openedition.org/jsa/12203

DOI : 10.4000/jsa. 12203

ISSN : $1957-7842$

\section{Éditeur}

Société des américanistes

\section{Édition imprimée}

Date de publication : 31 juillet 2012

Pagination : 201-206

ISSN : 0037-9174

\section{Référence électronique}

Nathalie Ragot, « LÓPEZ LUJÁN Leonardo y Guilhem OLIVIER (eds), El sacrificio humano en la tradición

religiosa mesoamericana ", Journal de la Société des américanistes [En ligne], 98-1 | 2012, mis en ligne le 01 septembre 2012, consulté le 04 septembre 2022. URL : http://journals.openedition.org/jsa/12203 ; DOI : https://doi.org/10.4000/jsa.12203

Ce document a été généré automatiquement le 4 septembre 2022

Tous droits réservés 


\title{
LÓPEZ LUJÁN Leonardo y Guilhem OLIVIER (eds), El sacrificio humano en la tradición religiosa mesoamericana
}

\author{
Nathalie Ragot
}

\section{RÉFÉRENCE}

LÓPEZ LUJÁN Leonardo y Guilhem OLIVIER (eds), El sacrificio humano en la tradición religiosa mesoamericana, Instituto nacional de Antropología e Historia/Universidad nacional autónoma de México, Mexico, 2010, 592 p., ill., photos

Ce gros livre de presque 600 pages est la publication des interventions d'un aréopage de 28 scientifiques internationaux (Mexicains, Belge, Français, Japonais, Canadiens, NordAméricains) réunis à Mexico en septembre 2007 pour un séminaire consacré au sacrifice humain. L'objectif principal de cet ouvrage est de présenter les nouvelles avancées sur les significations, les fonctions et l'évolution historique du sacrifice humain en Mésoamérique en considérant les informations émanant de diverses disciplines: archéologie, anthropologie physique, biologie, histoire des religions et ethnologie. Comme le souligne Alicia Mayer, directrice de l'Instituto de investigaciones históricas de la UNAM, dans l'introduction, «les fonctions et les significations du sacrifice humain sont nombreuses et varient en fonction des participants et des contextes rituels; cet événement ne peut être réduit à des interprétations univoques et il est nécessaire de l'analyser dans le cadre de ses différents contextes historiques » (p. 18). À cette fin, les interventions rendent compte des multiples expressions du sacrifice dans des géographies et à des époques différentes, allant du Préclassique à la période contemporaine, tout en couvrant la majeure partie du territoire mésoaméricain, des chaudes terres mayas du Sud aux arides terres du Nord.

Organisées en quatre grandes parties, les 26 contributions sont précédées de deux textes introductifs. Le premier, rédigé par les coordinateurs, Leonardo López Luján et Guilhem 
Olivier, présente un bref résumé des principales études dédiées au sacrifice humain en Mésoamérique depuis la fin du XIX ${ }^{e}$ siècle. S'y ajoute une précieuse synthèse des apports du livre El sacrificio humano en la tradición religiosa mesoamericana et des perspectives de recherche qu'il ouvre. Dans le second texte introductif, Eduardo Matos Moctezuma, après avoir démontré à l'aide d'exemples la présence du sacrifice humain dans les cultures antiques, établit un tableau général de l'immolation humaine en Mésoamérique. Il expose les principaux mythes fondateurs de l'occision rituelle (qui, en général, sont à l'origine de cette pratique sur la mort originelle des dieux) et les différentes formes qu'elle adoptait (cardiectomie, décapitation, noyade, égorgement, etc.).

3 La première partie de El sacrificio humano... est consacrée à l'« antiquité et [la] diversité du sacrifice humain en Mésoamérique» (p. 65). Elle réunit des textes qui donnent une vision plus ou moins générale de ce phénomène religieux à travers le vaste territoire mésoaméricain (zone maya, Nord, Michoacan, côte du Golfe, Oaxaca et Teotihuacan) sur une vaste échelle temporelle allant du Préclassique ancien au Postclassique récent. Selon les zones et les époques, les témoignages sont très variables, plus ou moins étudiés et publiés. Dans le premier article, Sara Ladrón de Guevara propose une révision critique des témoignages sur la présence de sacrifice sur la côte du Golfe. D'après cet auteur, dans le corpus des sculptures connues de la culture olmèque, rien ne permet, au niveau archéologique ou au niveau iconographique, de penser de façon évidente que le sacrifice ait existé, même si certains indices le laissent supposer. Après les Olmèques, les témoignages iconographiques univoques de sacrifices humains se multiplient, en particulier ceux ayant trait à la décollation. Que ce soit en association avec le jeu de balle pendant la période classique ou dans les dépôts mortuaires et avec le tzompantli au Postclassique, la décapitation est bien présente sur la côte du Golfe. À Teotihuacan, nombreux sont les témoignages archéologiques qui témoignent d'une activité sacrificielle établie dès la fondation de la ville à l'époque classique. L'article de Saburo Sugiyama fournit un panorama général des traces de sacrifices présentes dans les principaux édifices de la ville (pyramides du Serpent à Plumes, de la Lune et du Soleil). Notant qu'aucune association directe n'a été constatée entre les témoignages archéologiques de sacrifice humain et des sépultures spécifiques (royales notamment), il en conclut que les sacrifices humains étaient apparemment dédiés aux constructions et formaient partie intégrante des rituels de fondation. Les dirigeants de Teotihuacan manifestaient leur idéologie religieuse par les constructions monumentales et utilisaient le sacrifice humain pour consolider les structures sociopolitique et militaire du système. L'article suivant s'intéresse à la région de Oaxaca. Se fondant sur le modèle d'interprétation structuraliste d'Hubert et Mauss, Javier Urcid analyse un vaste ensemble de données disparates provenant des sphères archéologique, iconographique, ostéologique et historique. Il insiste sur le caractère fondamentalement substitutif du sacrifice humain et étudie son caractère polysémique à travers les notions de personne, de corps (utilisation des têtes coupées, des peaux, des restes osseux et du sang des sacrifiés), de communauté (sacrifice comme promoteur d'identité sociale), de lieu et de divinité. La riche contribution de Stephen Houston et d'Andrew Scherer rassemble de nombreux témoignages iconographiques, épigraphiques et ostéologiques rendant compte de la large diffusion du sacrifice humain dans la partie centrale de l'aire maya, le Petén, à l'époque classique. Textes et images informent sur les multiples manières d'immoler les victimes (lapidation, éviscération, cardiectomie, mise à mort par le feu, écorchement, décapitation) et de traiter leurs dépouilles (désarticulation, démembrement, passage par le feu, usage des crânes ou des mandibules comme 
trophées). Archéologie et ostéologie confirment souvent ces pratiques et mettent en lumière la complexité des rituels autour du sacrifice, pas toujours apparente dans l'iconographie. L'article de Vera Tiesler et Andrea Cucina poursuit l'étude de l'activité sacrificielle en zone maya, en s'intéressant aux pratiques des Mayas péninsulaires de la période classique à la Conquête. Ils distinguent deux grandes catégories de sacrifices pratiqués dans le Yucatan: les sacrifices de communion par lesquels les Mayas cherchaient à établir une communication avec les forces sacrées afin d'assurer la continuité et le bien-être de la communauté; les sacrifices de prestige où les mises à mort rituelles devaient bénéficier aux dirigeants. Élargissant la présentation des pratiques sacrificielles à travers la Méoamérique, Marie-Areti Hers offre une intéressante réflexion sur les relations entre le Nord, peuplé par les redoutés Tolteca-Chichimeca, et le haut plateau central. Elle rappelle l'hypothèse selon laquelle certains éléments liés au sacrifice humain, notamment le chacmol et le tzompantli, largement utilisés par les Toltèques et les Aztèques à l'époque postclassique, auraient une origine nordique datant de l'époque classique. Concluant cette première partie, Grégory Pereira propose une précise et précieuse étude de toutes les évidences du sacrifice humain chez les Tarasques d'après la Relación de Michoacán. Il identifie deux grandes catégories de sacrifices. La première est celle principalement des guerriers captifs, destinés aux divinités célestes et associés à la moitié supérieure du monde. La deuxième concerne plutôt les victimes issues de la communauté, les condamnés par la loi, considérés comme liés à l'impureté, mais aussi à la fertilité et mis à mort en l'honneur des divinités telluriques et du monde aquatique.

4 La deuxième partie de l'ouvrage réunit des textes consacrés uniquement aux Mexica selon l'archéologie et l'anthropologie physique. C'est sans aucun doute cette partie qui contient les données les plus novatrices, grâce aux découvertes faites, ces dernières décennies, suite à l'analyse de témoignages trouvés in situ. Le texte de Salvador Guilliem Arroyo, fondé sur les données de ses propres fouilles et sur les carnets de terrain de l'archéologue Francisco Gonzales Rul, est une synthèse des différents ensembles archéologiques (soit plusieurs tzompantli et des centaines d'inhumations contenant des dépouilles de victimes adultes et infantiles, des restes osseux mutilés et des sacrifices de fondation) témoignant de la pratique des sacrifices humains à Tlatelolco à l'époque postclassique. Il pose, avec raison, la question de l'étonnant contraste entre la variété et l'abondance des témoignages du sacrifice à Tlatelolco et sa relative rareté à Tenochtitlan. Avec les deux articles suivants, on entre pleinement dans le domaine de l'anthropologie physique. Carmen María Pijoán Aguadé et Josefina Mansilla Lory analysent, de façon minutieuse, des restes osseux humains de l'enceinte sacrée de Mexico-Tlatelolco, récupérés pendant les campagnes de fouilles des années 1960-1964. Ces deux auteurs révèlent, grâce à ce travail, les diverses traces d'origine rituelle sur les corps de sacrifiés et apportent de subtiles précisions, notamment sur certains aspects techniques de la cardiectomie. Ximena Chávez Balderas consacre sa contribution aux «modifications culturelles » (p. 318) perpétrées sur les corps des sacrifiés dont les têtes décapitées furent utilisées à des fins rituelles. Elle analyse en détail les 72 crânes découverts dans des offrandes du Templo Mayor de Tenochtitlan et les classe en trois catégories: les têtes tranchées déposées dans les offrandes de consécration et nommées " têtes-trophées "; les crânes féminins et masculins, décharnés et perforés aux tempes, qui étaient exposés sur le tzompantli; les crânes modifiés pour ne garder et décorer que la partie faciale, appelés « masques-crânes ». Juan Alberto Román Bellereza, quant à lui, s'intéresse au rôle des enfants dans la représentation du monde et les pratiques rituelles. Étudiant le 
matériel archéologique et les textes sur Tenochtitlan et Tlaltelolco, il propose plusieurs explications sur le choix des enfants comme victimes sacrificielles en fonction des contextes. Achevant cette seconde partie, le bel article écrit à quatre mains par Leonardo López Luján, Ximena Chávez Balderas, Norma Valentín et Aurora Montúfar, analyse en détail la remarquable découverte de l'offrande 111 au pied de la grande pyramide double du Templo Mayor. Située dans la section sud du sanctuaire, celle correspondant au culte de Huitzilopochtli, l'offrande 111 renfermait le squelette d'un enfant de 5 ans immolé par arrachement du cœur. Les ornements dont il était paré - notamment une paire d'ailes d'épervier - l'identifient comme étant un ixiptla, une « image », de Huitzilopochtli.

La troisième partie du volume est aussi consacrée aux Mexica, mais cette fois dans « la perspective de l'histoire» (p.395). Y sont donc réunis des travaux d'historiens qui abordent les fonctions et significations politiques, sociales et religieuses du sacrifice humain. L'article de Yolotl González Torres se penche sur les dimensions de domination et de soumission que le sacrifice humain a symbolisées à l'époque des derniers gouvernants tenochca. L'évolution historique des Mexica, qui a conduit à la formation de l'empire et l'instauration de la " guerre fleurie ", expliquerait l'augmentation du nombre des victimes humaines. Le sacrifice humain aurait ainsi été utilisé par la classe dirigeante comme moyen de soumission des populations vaincues ou tributaires. La contribution de Michel Graulich est une minutieuse analyse des différents lieux du sacrifice. Il détaille d'abord tous les lieux de mise à mort (montagnes, grottes, lagunes, grands temples et pyramides), puis les différents supports d'immolation et d'exposition des victimes (pierre de sacrifice, autels, tzompantli, chacmol, cuauhxicalli). De chacun, il étudie ensuite longuement les décorations et les symbolismes associés. Carlos Javier González González s'intéresse au sacrifice humain en tant que générateur de prestige et moteur de promotion sociale des guerriers tenochca. S'appuyant sur l'exemple de la fête de Tlacaxipehualiztli, il montre comment les guerriers, propriétaires des sacrifiés mis à mort dans ce contexte, acquéraient prestige, biens matériels et pouvoir au sein de la hiérarchie militaire mexica. Également consacré à Tlacaxipehualiztli, l'article de ClaudeFrançois Baudez développe une intéressante interprétation symbolique de certains aspects de cette fête. Partant de la comparaison entre les fêtes de Tlacaxipehualiztli et d'Ochpaniztli, qui mettent toutes les deux en scène l'écorchement des victimes, masculines pour la première, féminines pour la deuxième, il élabore une réflexion sur la fonction symbolique de l'écorchement qui symboliserait le passage d'une identité à une autre. Dans un bel article dédié aux divers symbolismes des Mimixcoa, Guilhem Olivier examine, pour sa part, les équivalences existant, dans l'imaginaire mexica, entre la guerre et la chasse. Tant dans les mythes que dans les rites, les Mimixcoa, prototypes des guerriers, sont traités comme des cerfs et les cerfs comme des guerriers, car les hommes et les cervidés constituent les victimes sacrificielles par excellence. Ce modèle de sacrifice étroitement lié à la guerre et à la chasse serait également à la base des rituels d'accession au pouvoir, en particulier les rites d'intronisation. La contribution de David Carrasco clôt cette partie en revenant sur la nécessité de comprendre le sacrifice humain, non seulement en fonction des conceptions mésoaméricaines de l'époque, où mythes et calendriers jouent un rôle essentiel, mais aussi en fonction des espaces, que ce soient ceux occupés par les instances du pouvoir ou tous ceux dévolus au sacrifice. Il s'interroge sur les relations entre la violence, le sacrifice et le prestige sacré de la cité dans les sociétés mésoaméricaines. Dans la continuité de cette réflexion, il présente plusieurs scènes de sacrifice figurées sur la carte de Cuauhtinchan II (Itzpapalotl sortant de Chicomoztoc et portant une pierre de sacrifice; offrande de cœur de sacrifié au soleil 
pendant la pérégrination ; sacrifice par des alliés des Mimixco d'une captive agenouillée ; sacrifice d'animaux, papillon, serpent, aigle, sauterelle dans le centre cérémoniel de Cholula).

6 La quatrième et dernière partie regroupe trois études ethnographiques consacrées aux Tlapanèques, aux Tzeltal et aux Huichol. Elles projettent un éclairage contemporain sur la pratique du sacrifice et son évolution depuis le passé préhispanique. Danièle Dehouve s'intéresse à la polysémie de l'immolation rituelle à travers l'analyse des sacrifices d'animaux pratiqués par les Tlapanèques du Guerrero. Ces immolations animales appartiennent à deux types de sacrifice distincts, lesquels devaient déjà exister à l'époque précolombienne. Le premier, le «sacrifice d'appropriation» (p.511), a pour objectif l'identification entre l'être humain et les puissances destinataires du sacrifice afin de s'emparer de leur force. Il concerne les animaux de la ferme - par exemple, la poule et les animaux domestiques puissants comme le chat et le chien. Le second, ou "sacrifice de séparation» (p.511), fait appel aux animaux non comestibles, comme le lézard ou la tarentule, et a pour but de se séparer de la puissance invoquée lors du rituel. Helios Figuerola Pujol expose plusieurs pratiques sacrificielles encore en vigueur chez les Tzeltal de Cancuc, au cours desquelles on substitue l'« âme» (p. 530) humaine par une " âme " animale. Sur un plan ontologique, la personne cancuc se compose de trois ch'ulel (la sombra, le ch'ulel ta ch'iibal, l'oiseau de cœur) et de nombreux lab qui peuvent être des humains, des animaux ou des phénomènes météorologiques. Les divinités se nourrissent des ch'ulel des présents rituels. Dans un contexte thérapeutique, l'acte sacrificiel consiste à échanger le ch'ulel capturé par celui de la victime sacrificielle. Dans les rituels prophylactiques ou ceux ayant trait à la fertilité le ch'ulel de la victime est offert à la terre pour obtenir ses faveurs. Dans les rituels à caractère nocif, le ch'ulel de l'animal sacrifié est assimilé au ch'ulel de la victime de la sorcellerie et donné à la Terre pour être dévoré. Johannes Neurath nous introduit enfin dans l'univers des Huichol et présente l'analyse des deux sortes de sacrifices qu'ils pratiquent: la chasse et le sacrifice d'animaux domestiques. Si les deux immolations ont de nombreux points communs, elles n'en sont pas moins opposées quant à leur signification symbolique et ésotérique. La chasse au cerf consiste à convaincre un cerf qu'il fasse un "autosacrifice » en se laissant mettre à mort par le chasseur, permettant ainsi la continuation de la coutume et de l'univers. La mise à mort d'animaux domestiques, tels que le bœuf ou le chevreau, qui rechignent à aller au sacrifice, est en relation avec la volonté de contrôler des aspects nocturnes chaotiques de la personne et du cosmos.

7 Pour terminer ce voyage à travers les multiples aspects du sacrifice mésoaméricain, un dernier article isolé présente, à titre comparatif, un cas andin. Steve Bourget, s'appuyant sur des données archéologiques et un riche corpus iconographique, cherche à comprendre les relations entre la pratique du sacrifice humain et les différentes formes d'autorités religieuses et politiques de la culture moche.

8 El sacrificio humano en la tradición religiosa mesoamericana est, on l'aura compris, une somme. C'est un ouvrage essentiel pour qui cherche à s'informer sur l'état des connaissances et des réflexions sur le thème du sacrifice humain dans les cultures mésoaméricaines. L'approche pluridisciplinaire, la variété des méthodologies et le large éventail des sociétés et des époques étudiées donnent une vision riche et multiple du sacrifice. Ce livre a également le très grand mérite de mettre à la disposition des lecteurs des éléments inédits issus de découvertes et d'avancées récentes, en particulier dans les domaines de l'archéologie, de la taphonomie et de l'ostéologie. Toutes ces qualités, 
assorties à une présentation claire, agréable et bien illustrée, font de l'ouvrage LA référence sur le sacrifice humain en Mésoamérique, un thème essentiel à l'échelle des sciences humaines en général.

\section{AUTEURS}

\section{NATHALIE RAGOT}

Université ouverte de Paris VII-Diderot 